\title{
Augmented eccentric connectivity index
}

\author{
Tomislav Došlić and Mahboubeh Saheli
}




\title{
AUGMENTED ECCENTRIC CONNECTIVITY INDEX
}

\author{
TOMISLAV DOŠLIĆ AND MAHBOUBEH SAHELI
}

Received February 16, 2011

\begin{abstract}
We review basic mathematical properties of the augmented eccentric connectivity index. Explicit formulas are presented for several classes of graphs, in particular for some open and closed unbranched polymers and nanostructures. Asymptotic behavior is explored and compression ratios are computed for those polymers.
\end{abstract}

2000 Mathematics Subject Classification: 05C12; 05C40; 05C90

Keywords: augmented eccentric connectivity index, distance based descriptor

\section{INTRODUCTION}

The eccentric connectivity index is one of topological invariants that have been recently used in the quantitative structure-activity relationship (QSAR) and quantitative structure-property relationship (QSPR) studies. Like many other useful invariants, it spawned several generalizations. One of them, the augmented eccentric connectivity index, turned out to be even better suited to certain tasks than the original invariant. In spite of its usefulness, its mathematical properties have never been investigated. The main goal of this paper is to review some basic mathematical properties of the augmented eccentric connectivity index and, where it is possible, to establish explicit formulas for certain classes of graphs. It turned out that those properties are very far from the properties of the original invariant and from the properties of other distancebased invariants such as, e.g., the Wiener number. We find that this is due to the combined effects of the non-local and non-linear nature of the vertex contributions.

\section{DEFINITIONS AND PRELIMINARIES}

All graphs in this paper are finite, simple and connected. For terms and concepts that are not defined here we refer the reader to any of the standard monographs such as, e.g., [7] or [13].

Let $G$ be a graph on $n$ vertices. We denote the vertex and the edge set of $G$ by $V(G)$ and $E(G)$, respectively. For two vertices $u$ and $v$ of $V(G)$ we define their distance $d(u, v)$ as the length of a shortest path connecting $u$ and $v$ in $G$. For a given vertex $u$ of $V(G)$ its eccentricity $\varepsilon(u)$ is the largest distance between $u$ and any other 
vertex $v$ of $G$. Hence, $\varepsilon(u)=\max _{v \in V(G)} d(u, v)$. The maximum eccentricity over all vertices of $G$ is called the diameter of $G$ and is denoted by $D(G)$; the minimum eccentricity among the vertices of $G$ is called radius of $G$ and is denoted by $R(G)$. The set of all vertices of minimum eccentricity is called the center of $G$.

The eccentric connectivity index $\xi(G)$ of a graph $G$ is defined as

$$
\xi(G)=\sum \delta_{u} \varepsilon(u)
$$

where $\delta_{u}$ denotes the degree of vertex $u$, i. e., the number of its neighbors in $G$. The eccentric connectivity index was introduced by Madan et al. and used in a series of papers concerned with QSAR/QSPR studies $[6,11,12]$. The study of its mathematical properties started only recently $[1-4,9,14]$; we refer the reader to a recent paper by Ilić [8] for a comprehensive survey.

The augmented eccentric connectivity index $A \xi(G)$ of a graph $G$ is defined as

$$
A_{\xi}(G)=\sum_{u \in V(G)} \frac{M(u)}{\varepsilon(u)}
$$

where $M(u)$ denotes the product of degrees of all neighbors of vertex $u$. It was introduced in another paper by the above mentioned group of authors [5]. We can see that the vertex contributions are both non-local (the degrees are taken over the neighborhoods and then multiplied) and non-linear in $\varepsilon(u)$. While the first fact is reflected in the name, the second one is completely ignored - it would have been better to call the new invariant something like augmented reciprocal eccentric connectivity index. The combination of those two properties makes the augmented eccentric connectivity index rather unyielding to the standard approach to distance-based invariants, as a number of difficulties arise when one tries to obtain explicit formulas or to find the extremal graphs and values.

A graph $G$ is vertex-transitive if its automorphism group is transitive. For a vertex-transitive graph $G$ its center coincides with $V(G)$. Since a vertex-transitive graph is necessarily regular, we have a particularly simple expression for the eccentric connectivity index of a vertex transitive graph.

Proposition 1. Let $G$ be a vertex-transitive graph on $n$ vertices of degree $\delta$. Then

$$
A_{\xi}(G)=\frac{n \delta^{\delta}}{R(G)}
$$

As a consequence, we obtain explicit formulas for the eccentric connectivity indices of several familiar classes of graphs.

Corollary 1.

(1) ${ }^{A} \xi\left(K_{n}\right)=n(n-1)^{n-1}$;

(2) ${ }^{A} \xi\left(C_{n}\right)=\frac{4 n}{\left\lfloor\frac{n}{2}\right\rfloor}=\left\{\begin{array}{ccc}8, & n & \text { even } \\ \frac{8 n}{n-1}, & n & \text { odd }\end{array}\right.$; 
(3) $A \xi\left(\Pi_{m}\right)=\frac{54 m}{\left\lfloor\frac{m}{2}\right\rfloor+1}=\left\{\begin{array}{lll}\frac{108 m}{m+2}, & m & \text { even } \\ \frac{108 m}{m+1}, & m & \text { odd }\end{array}\right.$;

(4) ${ }^{A} \xi\left(A_{m}\right)=\frac{512 m}{\left\lfloor\frac{m+1}{2}\right\rfloor}=\left\{\begin{array}{lcc}1024, & m & \text { even } \\ \frac{1024 m}{m+1}, & m & \text { odd }\end{array}\right.$;

(5) $A \xi\left(Q_{m}\right)=2^{m} m^{m-1}$.

Here $K_{n}, C_{n}, \Pi_{m}, A_{m}$ and $Q_{m}$ denote the complete graph on $n$ vertices, the cycle on $n$ vertices, the $m$-sided prism, the $m$-sided antiprism, and the $m$-dimensional hypercube, respectively.

It is immediately seen that the complete graph has the largest augmented eccentric connectivity index among all graphs on the same number of vertices. Namely, no vertex in a simple graph $G_{n}$ on $n$ vertices can contribute more than $(n-1)^{n-1}$ to $A_{\xi}\left(G_{n}\right)$, and the only graph on $n$ vertices in which each vertex contributes that much is $K_{n}$. Hence, for any graph $G_{n} \neq K_{n}$ on $n$ vertices we have ${ }^{A} \xi\left(G_{n}\right)<{ }^{A} \xi\left(K_{n}\right)$. The above result also shows that the upper bound on ${ }^{A} \xi\left(G_{n}\right)$ is super-factorial (i.e., it grows faster than the factorial function) in the number of vertices, in a sharp contrast to the polynomial behavior of the eccentric connectivity index. On the other hand, the result for the cycles shows that no lower bounds better than a constant can be established for general graphs. Hence, the augmented eccentric connectivity index can, at least in principle, attain its values in a very large span. This might partly explain and account for its observed low degeneracy.

The following results can be easily obtained by a straightforward computation.

Proposition 2. Let $W_{n}$ and $B_{n}$ denote the graphs of the pyramid and the bipyramid with $n$-gonal base, $n \geq 3$. Then

$$
A_{\xi}\left(W_{n}\right)=3^{n}+\frac{9}{2} n^{2} \quad \text { and } \quad A_{\xi}\left(B_{n}\right)=4^{n}+8 n^{3} .
$$

(The pyramid graph $W_{n}$ is also known as the wheel graph on $n$ spokes.)

Proposition 3. Let $K_{m, n}$ be a complete bipartite graph on $m+n$ vertices. For $m, n \geq 2$, we have

$$
A_{\xi}\left(K_{m, n}\right)=\frac{1}{2}\left(m^{n+1}+n^{m+1}\right) .
$$

The case of the complete bipartite graph $K_{m, n}$ when one of the classes of bipartition is of size 1 brings us to the trees, hence it is treated separately. In order to facilitate the comparison with other trees on $n$ vertices, we find it more convenient to state the result as follows.

Proposition 4. Let $S_{n}=K_{1, n-1}$ be a star on $n \geq 3$ vertices. Then

$$
A_{\xi}\left(S_{n}\right)=\frac{(n-1)^{2}}{2}+1 \text {. }
$$




\section{LINEAR vs. CIRCULAR POLYMERS AND THE COMPRESSION RATIO}

It is intuitively clear that a typical distance-based graph invariant (such as the eccentric connectivity index or the Wiener index) of an open linear polymer should exceed the value of the same invariant for a closed polymer of the same size and type. That idea has been quantified by introducing the concept of compression ratio in [1], where it was shown that the eccentric connectivity index reduces by a factor of $2 / 3$ when an open polymer is closed by joining its end units, for a large enough number of units. Let $G_{n}^{o}$ be an open linear polymer built from $n$ identical units joined in a certain way, and $G_{n}^{c}$ be a closed linear polymer obtained from $G_{n}^{o}$ by identifying the free parts of its end units. For a graph invariant $i(G)$, its compression ratio is the limit of the quotient $i\left(G_{n}^{c}\right) / i\left(G_{n}^{o}\right)$ when $n \longrightarrow \infty$. For example, it can be easily verified that the compression ratio for the Wiener numbers of paths and cycles is $3 / 4$.

We will show in this section that the augmented eccentric connectivity index behaves exactly in the opposite way: its values for open polymers tends to be smaller than for their closed analogues. Hence we obtain "compression ratios" larger than one.

The simplest linear polymers are paths and cycles, and we start with them.

\subsection{Paths vs. cycles}

We start by recalling that the $n$-th harmonic number $H_{n}$ is given as the $n$-th partial sum of the harmonic series, $H_{n}=\sum_{i=1}^{n} \frac{1}{i}$. Since the eccentricities of vertices of a path increase by one from $\lceil n / 2\rceil$ to $n$, and the products of degrees of their neighborhoods are fairly constant, the following formula can be verified by a direct computation. Here $P_{n}$ denotes the path of length $n$, hence on $n+1$ vertices.

Proposition 5. Let $P_{n}$ be a path of length $n \geq 3$. Then ${ }^{A} \xi\left(P_{n}\right)=8\left(H_{n}-H_{\lfloor n / 2\rfloor}\right)-$ $R_{n}$, where

$$
R_{n}=\left\{\begin{array}{ll}
\frac{4}{n(n-1)}, & n \text { even } \\
\frac{8 n-4}{n(n-1)}, & n \text { odd }
\end{array} .\right.
$$

It is well known that harmonic numbers asymptotically behave as $H_{n} \approx \ln n+\gamma$, where $\gamma$ is the Euler-Mascheroni constant. Hence, ${ }^{A} \xi\left(P_{n}\right)$ is asymptotically equal to $8 \ln 2$. As a corollary, we obtain the compression ratio of the augmented eccentric connectivity index for paths and cycles.

Corollary 2.

$$
\lim _{n \longrightarrow \infty} \frac{A \xi\left(C_{n}\right)}{A \xi\left(P_{n}\right)}=\frac{1}{\ln 2} .
$$

\subsection{Ladder graphs vs. prisms}

Let $L_{n}$ be a ladder graph, i.e, a graph obtained as the Cartesian product of $P_{n}$ and $K_{2}$. It has $n$ squares, as shown in Fig. 1. It can be verified by direct computation that 


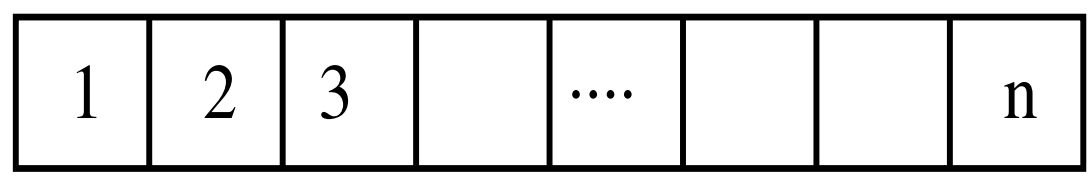

FIGURE 1. A ladder graph.

for even $n$ the following formula is valid.

$$
A_{\xi}\left(L_{n}\right)=108\left(H_{n}-H_{n / 2}\right)-\frac{36}{n}+\frac{24}{n+1}-\frac{108}{n+2} .
$$

For an odd $n$ the formula differs only in the correction terms. Both cases can be expressed by a single formula.

Proposition 6.

$$
A_{\xi}\left(L_{n}\right)=108\left(H_{n}-H_{\lceil n / 2\rceil}\right)-\frac{36}{n}+\frac{24}{n+1}-\frac{1+(-1)^{n}}{2} \frac{108}{n+2} .
$$

Asymptotically, both cases behave like $108 \ln 2$. Since the closed version of $L_{n}$ is the prism $\Pi_{n}$, we obtain again $\frac{1}{\ln 2}$ as the compression ratio.

\subsection{Hexagonal chains vs. hexagonal belts}

In this subsection $Z_{h}^{o}$ and $A_{h}^{o}$ denote an open zigzag and an open armchair chain of $h$ hexagons, respectively. They are shown in Figs 2 and 3. The corresponding<smiles>C1CCC2CC3CC4CC5CC6CC7CCCCC7CC6CC5CC4CC3CC2C1</smiles>

FIGURE 2. A zigzag hexagonal chain.

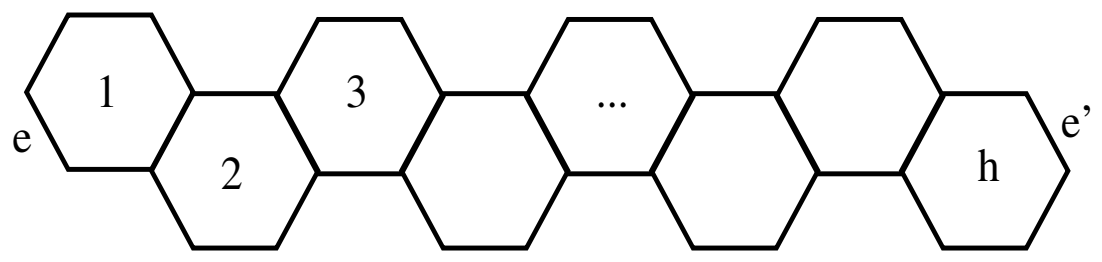

FIGURE 3. An armchair hexagonal chain.

closed hexagonal polymers are obtained in both cases by identifying the edges $e$ and 
$e^{\prime}$. They are denoted by $Z_{h}^{c}$ and $A_{h}^{c}$ and called the zigzag and the armchair hexagonal belt, respectively. We start with belts, since their augmented eccentric connectivity indices are easier to compute.

In the case of zigzag belts there are $4 h$ vertices; all of them have the eccentricity equal to $h+1$. For $2 h$ of them the product of degrees of their neighbors is equal to 9 , for another $2 h$ of them it is equal to 12 . By summing all contributions we readily obtain $A \xi\left(Z_{h}^{c}\right)=42 \frac{h}{h+1}$.

The armchair belt $A_{h}^{c}$ also has $4 h$ vertices. For $h>4$ (note that $h$ must be even!) all of them have the eccentricity equal to $h$. By summing the contributions of two types of vertices we obtain $A \xi\left(A_{h}^{c}\right)=2 h\left(\frac{6}{h}+\frac{18}{h}\right)=48$. For the exceptional case $h=4$ we have ${ }^{A} \xi\left(A_{4}^{c}\right)=\frac{228}{5}$.

Now we consider open chains. The case of the armchair chain is simpler, in the sense that the formula does not depend on the parity of $h$. By ordering the contributions of vertices by increasing eccentricity we obtain

$$
\begin{aligned}
A_{\xi}\left(A_{h}^{o}\right) & =2\left(\sum_{i=1}^{h-1} \frac{6}{h+i}+\sum_{i=2}^{h-1} \frac{18}{h+i}+\frac{4}{2 h}+\frac{6}{2 h}+\frac{4}{2 h+1}\right) \\
& =48 H_{2 h}-48 H_{h}-2 \frac{46 h^{2}+35 h+7}{h(h+1)(2 h+1)} .
\end{aligned}
$$

For large values of $h$ this behaves like $48 \ln 2$, and by dividing $A \xi\left(A_{h}^{c}\right)=48$ by this value we obtain the expected compression ratio of $\frac{1}{\ln 2}$.

For the zigzag chain we must consider two cases. For an even $h$ there are two vertices of degree 3 on the symmetry axis (and hence with the smallest eccentricity of $h+1)$ and their joint contribution to $A \xi\left(Z_{h}^{o}\right)$ is equal to $\frac{24}{h+1}$. All other classes of vertices with the same eccentricity have four elements, and the eccentricities increase from $h+2$ to $2 h+1$. However, the resulting sum cannot be neatly expressed in terms of harmonic numbers, due to the fact that the numerators of the contributions from the vertices with an even eccentricity are not the same as of those with odd eccentricities. Instead, the digamma function $\psi(z)=\Gamma^{\prime}(z) / \Gamma(z)$ appears, and we obtain

$$
A_{\xi}\left(Z_{h}^{o}\right)=36\left(H_{2 h}-H_{h}\right)+6\left(\psi\left(h+\frac{1}{2}\right)-\psi\left(\frac{h+3}{2}\right)\right)+2 \frac{26 h^{2}+11 h-3}{h(h+1)(2 h+1)}
$$

(The use of digamma function is not essential for obtaining our results, but it allows us to present them in a more compact way.) It can be checked by direct calculation that the above quantity behaves as $42 \ln 2$ for large values of $h$, and again we obtain the expected compression ratio $\frac{1}{\ln 2}$. The case of odd $h$ is similar and we omit the details. 


\subsection{Nanotubes vs. nanotori}

We start by considering a hexagonal network such as the one shown in Fig. 4. Its odd columns are made of $r$ hexagons, while the even ones have $r-1$ hexagons. By wrapping the network around its vertical symmetry axis so that the leftmost and the rightmost vertex of the same row are connected by an edge, we obtain a nanotube with $r$ rows and $2 s$ columns of hexagons. Such a nanotube is called an armchair nanotube and denoted by $N(r, s)$. We note that in an armchair nanotube the number

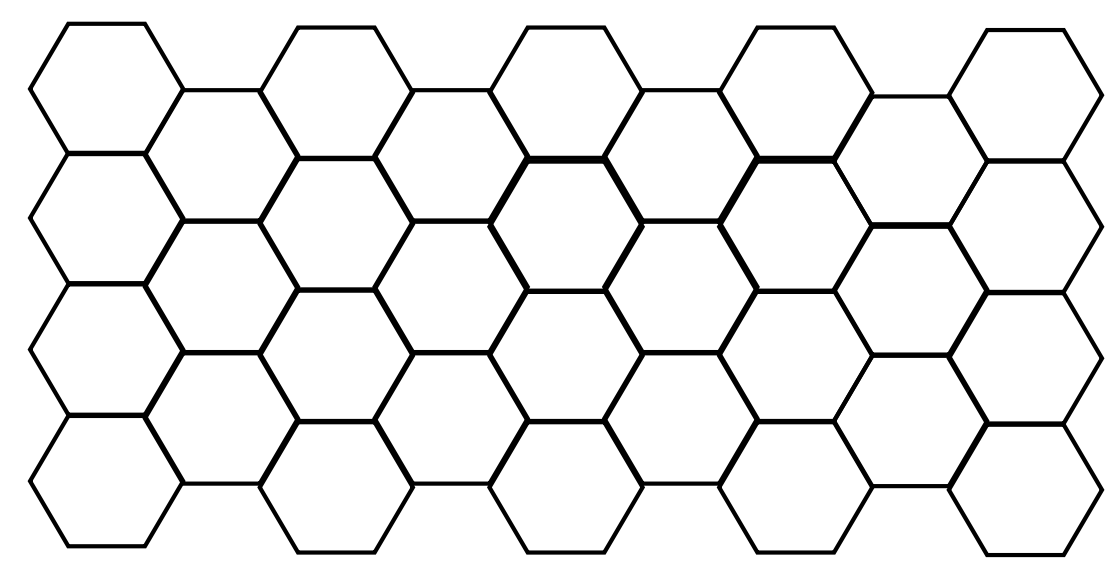

FIGURE 4. An armchair nanotube $(N(4,5))$.

of columns is always even. For the sake of simplicity we restrict our attention only to the armchair nanotubes that have a horizontal plane of symmetry.

The distances (and hence the eccentricities) relevant for our purpose were computed in a paper dealing with the eccentric connectivity index of armchair nanotubes [10]. By using those results we can derive explicit formulas for ${ }^{A} \xi(N(r, s))$ in terms of $r$ and $s$. The exact expressions depend on the relationship between those two parameters. For example, when $r$ is small $(2 r \leq s)$, all vertices have the same eccentricity and it is equal to $2 s$. There are $4 s$ vertices of degree 2 whose neighbors have degrees 2 and 3 . Hence their collective contribution is equal to $4 s \cdot 2 \cdot 3 /(2 s)=12$. Further, there are $4 s$ vertices of degree 3 whose neighbors contribute $2 \cdot 3 \cdot 3$ to the numerators, and their collective contribution is equal to $4 s \cdot 18 /(2 s)=36$. Finally, all other $(2 r+1) 2 s-8 s=4 r s-6 s$ vertices contribute $27 /(2 s)$ each, making it altogether $54 r-81$. By adding the contributions from all types of vertices we obtain that for small $r$ the augmented eccentric connectivity index of $N(r, s)$ is given by $54 r-33$. Since the other computations involved are similarly straightforward, we omit the details. 
Proposition 7. The values of ${ }^{A} \xi(N(r, s))$ are given by following formulas:

$$
\begin{array}{ll}
54 r-33 & , \text { for } 2 r \leq s ; \\
108 s\left(H_{2 r+s}-H_{2 s}\right)+27(2 s-2 r+1)-\frac{84 s}{2 r+s}-\frac{36 s}{2 r+s-1} & , \text { for } s<2 r<2 s \\
108 s\left(H_{2 r+s}-H_{r+s-3}\right)-\frac{54 s}{r+s}-\frac{84 s}{2 r+s}-\frac{36 s}{2 r+s-1} & , \text { for } r \geq s .
\end{array}
$$

Again, for large values of $r$ we can observe the familiar logarithmic behavior of $A_{\xi}(N(r, s))$ with respect to $r$.

From an armchair nanotube we can make an armchair nanotorus $T(r, s)$ by identifying the uppermost and the lowermost horizontal edges in each column of $r$ hexagons. When $r$ is large enough $(r \geq s)$ the eccentricity of all its vertices will be given by $r+s$. All vertices are now of degree 3, hence all numerators are equal to 27, and the augmented eccentric connectivity index of $T(r, s)$ is given by $A \xi(T(r, s))=(4 r s+2 s) \frac{27}{r+s}$. For large enough values of $r$ it behaves asymptotically as $108 s$. By considering the quotient ${ }^{A} \xi(T(r, s)) /{ }^{A} \xi(N(r, s))$ we again recover the familiar compression ratio of $1 / \ln 2$.

Similar results can be derived also for zigzag nanotubes and nanotori.

\section{CONCLUDING REMARKS}

In this paper we have presented some basic mathematical properties of the augmented eccentric connectivity index. However, our results still leave much to be desired. For example, we did not find the minimum value of ${ }^{A} \xi(G)$ over all graphs on a given number of vertices. We are convinced that this value is achieved for $P_{n-1}$, but the conjecture did not yield to the usual techniques. Further, it would be interesting to determine for which graphs $G$ their augmented eccentric connectivity index remains bounded. We have seen that $A \xi(G)$ can grow very fast with the number of vertices. As of now, we do not know for which classes of graphs the growth rate remains polynomial.

Acknowledgment. Partial support of the Ministry of Science, Education and Sport of the Republic of Croatia (Grants No. 037-0000000-2779 and 177-0000000-0884) is gratefully acknowledged by one of the authors (TD).

\section{REFERENCES}

[1] T. Došlić, A. Graovac, and O. Ori, "Eccentric connectivity index of hexagonal belts and chains," MATCH Commun. Math. Comput. Chem., vol. 65, no. 3, pp. 745-752, 2011.

[2] T. Došlić, A. Loghman, and L. Badakshian, "Computing topological indices by pulling a few strings," MATCH Commun. Math. Comput. Chem., vol. 67, no. 1, pp. 173-190, 2012.

[3] T. Došlić and M. Saheli, "Eccentric connectivity index of benzenoid graphs," in Novel Molecular Structure Descriptors - Theory and Applications II, I. Gutman and B. Furtula, Eds. Kragujevac: Univ. Kragujevac, 2010, pp. 169-182.

[4] T. Došlić, M. Saheli, and D. Vukičević, "Eccentric connectivity index: extremal graphs and values," Iranian J. Math. Chem., vol. 1, pp. 45-56, 2010. 
[5] H. Dureja and A. K. Madan, "Superaugmented eccentric connectivity indices: new-generation highly discriminating topological descriptors for QSAR/QSPR modeling," Med. Chem. Res., vol. 16, pp. 331-341, 2007.

[6] S. Gupta, M. Singh, and A. K. Madan, "Application of graph theory: relationship of eccentric connectivity index and Wiener's index with anti-inflammatory activity," J. Math. Anal. Appl., vol. 266, no. 2, pp. 259-268, 2002.

[7] F. Harary, Graph theory. Reading, Mass.-Menlo Park, Calif.-London: Addison-Wesley Publishing Co., 1969.

[8] A. Ilić, "Eccentric connectivity index," in Novel Molecular Structure Descriptors - Theory and Applications II, I. Gutman and B. Furtula, Eds. Kragujevac: Univ. Kragujevac, 2010, pp. 139168

[9] A. Ilić and I. Gutman, "Eccentric connectivity index of chemical trees," MATCH Commun. Math. Comput. Chem., vol. 65, no. 3, pp. 731-744, 2011.

[10] M. Saheli and A. R. Ashrafi, "The eccentric connectivity index of armchair polyhex nanotubes," Maced. J. Chem. Chem. Eng., vol. 29, pp. 71-75, 2010.

[11] S. Sardana and A. K. Madan, "Application of graph theory: relationship of molecular connectivity index, Wiener's index and eccentric connectivity index with diuretic activity," Match, no. 43, pp. 85-98, 2001.

[12] V. Sharma, R. Goswami, and A. K. Madan, "Eccentric connectivity index: A novel highly discriminating topological descriptor for structure-property and structure-activity studies," J. Chem. Inf. Comput. Sci., vol. 37, pp. 273-282, 1997.

[13] D. B. West, Introduction to graph theory. Upper Saddle River, NJ: Prentice Hall, Inc., 1996.

[14] B. Zhou and Z. Du, "On eccentric connectivity index," MATCH Commun. Math. Comput. Chem., vol. 63, no. 1, pp. 181-198, 2010.

Authors' addresses

Tomislav Došlić

University of Zagreb, Faculty of Civil Engineering, Kačićeva 26, 10000 Zagreb, Croatia

E-mail address: doslicegrad.hr

\section{Mahboubeh Saheli}

University of Kashan, Faculty of Sciences, 87317-51167 Kashan, Iran

E-mail address: mahboubeh. saheli@gmail.com 